\title{
Efficient conversion of xylose to ethanol by stress-tolerant Kluyveromyces marxianus BUNL-21
}

\author{
Sukanya Nitiyon' ${ }^{1}$, Chansom Keo-oudone ${ }^{2}$, Masayuki Murata' ${ }^{1}$, Noppon Lertwattanasakul ${ }^{3}$, Savitree Limtong ${ }^{3}$, \\ Tomoyuki Kosaka ${ }^{4}$ and Mamoru Yamada ${ }^{1,4^{*}}$
}

\begin{abstract}
The fermentation ability of thermotolerant Kluyveromyces marxianus BUNL-21 isolated in Laos was investigated. Comparison with thermotolerant K. marxianus DMKU3-1042 as one of the most thermotolerant yeasts isolated previously revealed that the strain possesses stronger ability for conversion of xylose to ethanol, resistance to 2-deoxyglucose in the case of pentose, and tolerance to various stresses including high temperature and hydrogen peroxide. K. marxianus BUNL-21 was found to have ethanol fermentation activity from xylose that is slightly lower and much higher than that of Scheffersomyces stipitis (Pichia stipitis) at $30^{\circ} \mathrm{C}$ and at higher temperatures, respectively. The lower ethanol production seems to be due to large accumulation of acetic acid. The possible mechanism of acetic acid accumulation is discussed. In addition, it was found that both K. marxianus strains produced ethanol in the presence of $10 \mathrm{mM}$ hydroxymethylfurfural or furfural, at a level almost equivalent to that in their absence. Therefore, K. marxianus BUNL-21 is a highly competent yeast for high-temperature ethanol fermentation with lignocellulosic biomass.
\end{abstract}

Keywords: Kluyveromyces marxianus, Thermotolerant yeast, Ethanol fermentation on xylose, Stress resistance

\section{Background}

Compared with Saccharomyces cerevisiae, which is widely used in ethanol fermentation industries, Kluyveromyces marxianus has advantageous potentials for application in ethanol production. First, $K$. marxianus is thermotolerant and is able to efficiently produce ethanol at high temperatures (Limtong et al. 2007), allowing us to develop high-temperature fermentation technology that will reduce cooling cost, enable efficient simultaneous saccharification and fermentation, reduce the risk of contamination and enable stable fermentation even in tropical countries (Anderson et al. 1986; Banat et al. 1998; Limtong et al. 2007). Second, the yeast can assimilate various sugars including xylose, arabinose, sucrose, raffinose and inulin in addition to several hexoses (Lertwattanasakul et al. 2011; Rodrussamee et al. 2011). This

\footnotetext{
*Correspondence: m-yamada@yamaguchi-u.ac.jp

${ }^{4}$ Department of Biological Chemistry, Faculty of Agriculture, Yamaguchi

University, Yamaguchi 753-8515, Japan

Full list of author information is available at the end of the article
}

broad spectrum in sugar assimilation capability is very beneficial for conversion of biomass consisting of various sugars to ethanol. Third, the yeast exhibits a relatively weak glucose repression on utilization of some sugars including sucrose (Lertwattanasakul et al. 2011) and is thus highly suitable for biomass such as sugar cane juice including glucose, fructose and sucrose as main sugars.

For application of such beneficial characteristics, however, some crucial points regarding $K$. marxianus should be improved. One point is a relatively strong glucose repression on utilization of other sugars including xylose (Rodrussamee et al. 2011). Another point is its low conversion ability of xylose to ethanol. The ethanol fermentation potential and sugar utilization profile of thermotolerant $K$. marxianus DMKU3-1042 have been extensively studied (Limtong et al. 2007; Lertwattanasakul et al. 2011; Rodrussamee et al. 2011; Pimpakan et al. 2012). The strain is capable of producing ethanol from all sugar components in lignocelluloses except arabinose. Lignocellulose, which is a second-generation biomass for biofuels, contains glucose, xylose and arabinose

\section{㩏 Springer}


as abundant sugars. Tremendous exploration (Toivola et al. 1984; Barnett et al. 2000; Kurtzman et al. 2011) has revealed that only a few yeast species can efficiently ferment D-xylose to ethanol. Xylose fermentation ability of $K$. marxianus strains isolated previously, however, is much lower than those of Scheffersomyces stipitis (Pichia stipitis) and Spathaspora passalidarum (Nguyen et al. 2006; Krahulec et al. 2012; Su et al. 2015), and the yield of ethanol production varies in different strains (Margaritis and Bajpai 1982; Banat et al. 1996; Wilkins et al. 2008; Rodrussamee et al. 2011). Gene engineering has thus been attempted to improve the ethanol productivity from xylose in K. marxianus (Wang et al. 2013; Zhang et al. 2013). In addition, it is known that toxic compounds, including hydroxymethylfurfural (HMF) and furfural, that are generated in the process of hydrolysis of lignocellulosic materials prevent the growth or fermentation efficiency of microbes (Mussatto and Roberto 2004; Behera et al. 2014), and strains that are resistant to them should thus be developed. It is notable that some ethanol-fermenting microbes such as S. stipitis are resistant to furfural at low concentrations (Nigam 2001).

In this study, we characterized a newly isolated strain of K. marxianus, BUNL-21 strain, which is one of the most thermotolerant yeasts screened in Laos. The BUNL-21 strain was shown to be superior to the DMKU3-1042 strain, which is one of the most thermotolerant and efficient $K$. marxianus strains isolated previously, in terms of the conversion activity of xylose to ethanol, resistance to 2-deoxyglucose in the case of pentose and tolerance to various stresses. In addition, we found efficient conversion of xylose to ethanol in the presence of HMF or furfural in $K$. marxianus strains tested. We also noticed a large accumulation of acetic acid on the xylose medium, which was more than that of ethanol, and we discuss its accumulation in comparison with $S$. stipitis.

\section{Methods}

Yeast strains

The yeast strains used in this study were thermotolerant K. marxianus BUNL-21 (NITE P-01739) and DMKU31042 (NITE AP-283), which were isolated in Lao PDR (Results for its screening and identification will be published elsewhere.) and Thailand (Limtong et al. 2007), respectively, and S. stipitis CBS 5773. They were stored in YPD medium (10 g/l yeast extract, $20 \mathrm{~g} / \mathrm{l}$ peptone and $20 \mathrm{~g} / \mathrm{l}$ glucose) supplemented with $10 \%(\mathrm{v} / \mathrm{v})$ glycerol at $-80{ }^{\circ} \mathrm{C}$.

\section{Analysis of ethanol fermentation ability}

To investigate the ethanol fermentation ability of $K$. marxianus strains, YP medium (10 g/l yeast extract and $20 \mathrm{~g} / \mathrm{l}$ peptone) supplemented with $20 \mathrm{~g} / \mathrm{l}$ of glucose (Glc) or xylose (Xyl), designated as YPD or YPXyl, respectively, was used. When mixed sugars were used, YP media supplemented with 2 or $20 \mathrm{~g} / \mathrm{l} \mathrm{Glc}$ and $20 \mathrm{~g} / \mathrm{l}$ of Xyl, designated as YPXyl $+0.2 \%$ Glc and YPXyl $+2 \%$ Glc, respectively, were used. Cells were precultured in $30 \mathrm{ml}$ of YPXyl or YPD medium in a 100-ml Erlenmeyer flask sealed with aluminium foil at $30{ }^{\circ} \mathrm{C}$ under a rotary shaking condition at $160 \mathrm{rpm}$ for $18 \mathrm{~h}$. The preculture was inoculated to $30 \mathrm{ml}$ fresh medium of YPXyl or YPD in a 100-ml Erlenmeyer flask sealed with aluminium foil at the initial $\mathrm{OD}_{660}$ value of 0.1 and incubated at 30 or $37{ }^{\circ} \mathrm{C}$ under a shaking condition at $160 \mathrm{rpm}$. The closure with aluminum foil may reduce oxygen transfer to flasks (Chain and Gualandi 1954) and cause an oxygen-limited condition. Yeast growth was determined by measuring optical density at $660 \mathrm{~nm}$ on a UVmini-1240 spectrophotometer (Shimadzu, Japan) and the values were converted to dry cell weight (DCW, g/l) by using conversion factors in equations in Additional file 1: Fig. S1. To determine sugar and ethanol concentrations in culture media, cultures were sampled and centrifuged at 14,000 rpm for 5 min. The supernatant was then subjected to quantitative analysis of sugars on a high-performance liquid chromatography apparatus (Hitachi, Japan) with a GLC610-S Gel pack column (Hitachi) connected to a refractive index detector Model L-2490 (Hitachi) in the mode of $0.5 \mathrm{ml} / \mathrm{min}$ eluent of deionized water at $60^{\circ} \mathrm{C}$. Acetic acid was analyzed on a high-performance liquid chromatography apparatus (Hitachi) with a GLC610-H Gel pack column (Hitachi) connected to a UV detector Model L-2400 (Hitachi) in the mode of $1 \mathrm{ml} / \mathrm{min}$ eluent of $0.1 \%$ phosphoric acid at $60{ }^{\circ} \mathrm{C}$. Ethanol concentration was analyzed by a gas chromatography GC-2014 apparatus (Shimadzu) with a glass column packed with PEG-20M (Shimadzu) connected to a flame ionization detector. We performed each experiment at least three times and obtained similar results.

\section{Analysis of stress resistance and glucose repression}

Cells were grown at $30{ }^{\circ} \mathrm{C}$ for $18 \mathrm{~h}$ in YPD medium, harvested, washed with distilled water, and resuspended in distilled water $\left(1 \times 10^{7}\right.$ cells $\left./ \mathrm{ml}\right)$. The cells were then tenfold sequentially diluted and spotted onto YPD agar plates supplemented with $5 \mathrm{mM}$ hydrogen peroxide $\left(\mathrm{H}_{2} \mathrm{O}_{2}\right), 8 \%(\mathrm{w} / \mathrm{v})$ ethanol, $35 \%(\mathrm{w} / \mathrm{v})$ Glc, $10 \mathrm{mM}$ furfural or $10 \mathrm{mM} \mathrm{HMF}$. The plates were incubated at $30{ }^{\circ} \mathrm{C}$ for $48 \mathrm{~h}$. To examine glucose repression on the assimilation of other sugars, 2-deoxyglucose (2-DOG) as a glucose analog was used. Cells were spotted onto YP agar plates with or without $0.01 \% 2-D O G$ in the presence of different carbon sources: $20 \mathrm{~g} / \mathrm{l}$ mannose (YPMan), $20 \mathrm{~g} / \mathrm{l}$ galactose (YPGal), $20 \mathrm{~g} / \mathrm{l}$ xylose (YPXyl) and $20 \mathrm{~g} / \mathrm{l}$ arabinose (YPAra). These plates were incubated at 30 or $37^{\circ} \mathrm{C}$ 
for $48 \mathrm{~h}$. We performed each experiment at least three times and obtained similar results.

\section{Results}

\section{Comparison of ethanol fermentation ability on Xyl} in K. marxianus BUNL-21 with those in K. marxianus DMKU3-1042 and S. stipitis at different temperatures Comparison of several $K$. marxianus strains allowed us to select BUNL-21 as the highest ethanol fermenting strain on Xyl. Its growth and ethanol fermentation ability were thus compared with those of the DMKU3-1042 strain, which is one of the most efficient strains that has been analyzed in detail (Limtong et al. 2007; Rodrussamee et al. 2011). In the case of Xyl as a carbon source, its assimilation and ethanol fermentation of DMKU3-1042 have been shown to be greatly reduced at high temperatures (Rodrussamee et al. 2011). Ethanol fermentation of DMKU3-1042 under a temperature-uncontrolled condition was performed, and the maximum temperature for the fermentation process was found to be $35^{\circ} \mathrm{C}$ (Murata et al. 2015). We thus compared the effects of temperatures $\left(30\right.$ and $\left.37^{\circ} \mathrm{C}\right)$ on ethanol fermentation from Xyl in BUNL-21, DMKU3-1042 and S. stipitis (Fig. 1; Table 1). At $30{ }^{\circ} \mathrm{C}$, biomass yields of BUNL-21 and S. stipitis were found to be lower than that of DMKU3-1042 in YPXyl medium. S. stipitis completely consumed Xyl within $48 \mathrm{~h}$, but a trace amount of Xyl remained in both $K$. marxianus strains. The maximum ethanol yields of BUNL-21, DMKU3-1042 and S. stipitis were 0.15, 0.09 and $0.31 \mathrm{~g} / \mathrm{g}$, respectively. At $37{ }^{\circ} \mathrm{C}$, S. stipitis could neither grow nor produce ethanol, but both $K$. marxianus strains grew well at this temperature. The time required to reach the maximum ethanol level was shorter, but the maximum ethanol yields were slightly lower than those at $30{ }^{\circ} \mathrm{C}$. BUNL-21 and DMKU3-1042 showed maximum ethanol yields of 0.14 and $0.07 \mathrm{~g} / \mathrm{g}$, respectively, and they completely consumed Xyl within $48 \mathrm{~h}$.

During xylose utilization, xylitol was accumulated in both $K$. marxianus strains but not in S. stipitis. Interestingly, there was little accumulation of xylitol in BUNL21 compared to that in DMKU3-1042 at $30{ }^{\circ} \mathrm{C}$, which seems to be consistent with a relatively small amount of ethanol in the latter, but the xylitol concentration significantly increased in both $K$. marxianus strains at $37{ }^{\circ} \mathrm{C}$. In addition, a large amount of acetic acid accumulated in both strains compared to that in S. stipitis, suggesting that the relatively low level of ethanol production in K. marxianus strains may be due to a high level of acetic

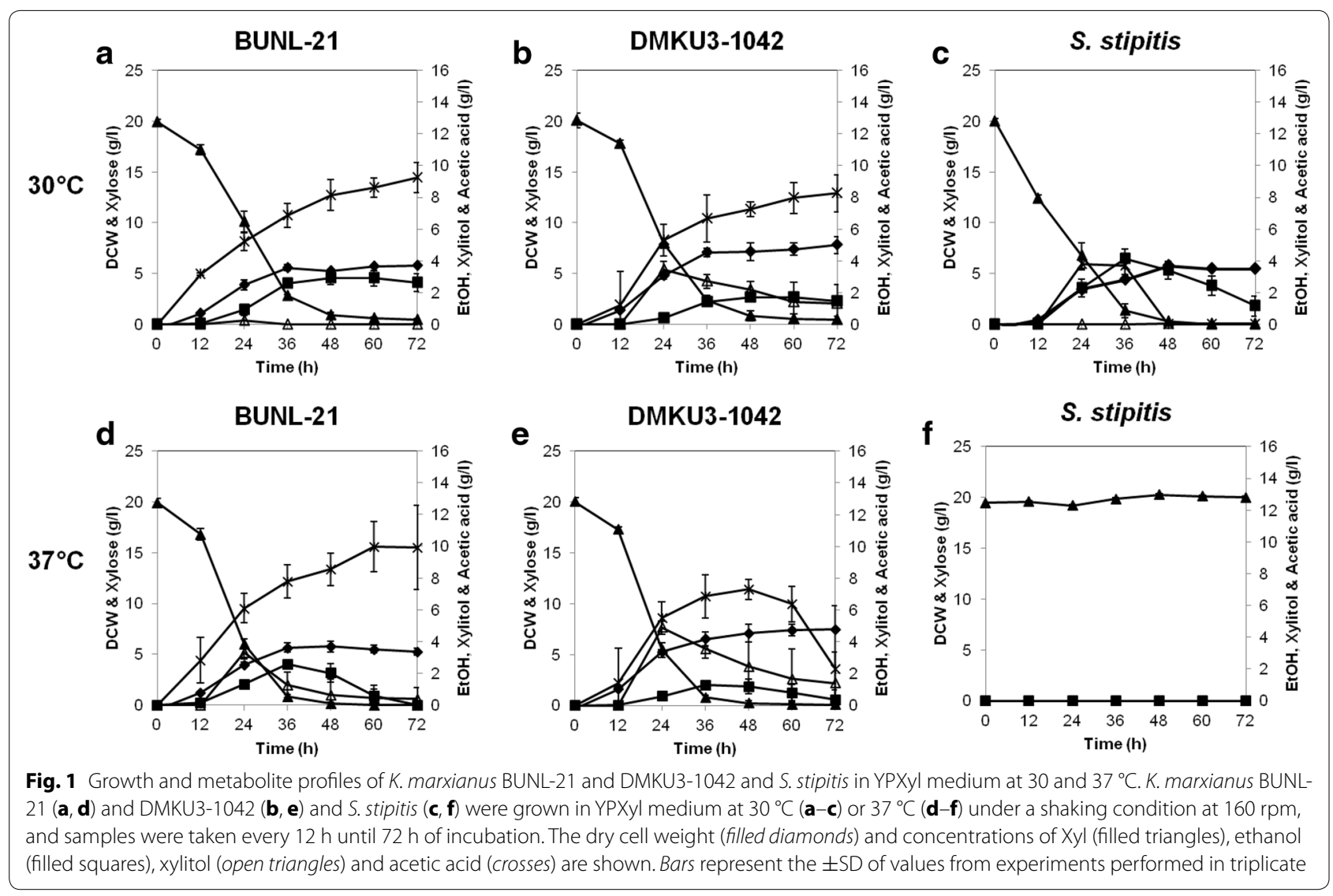




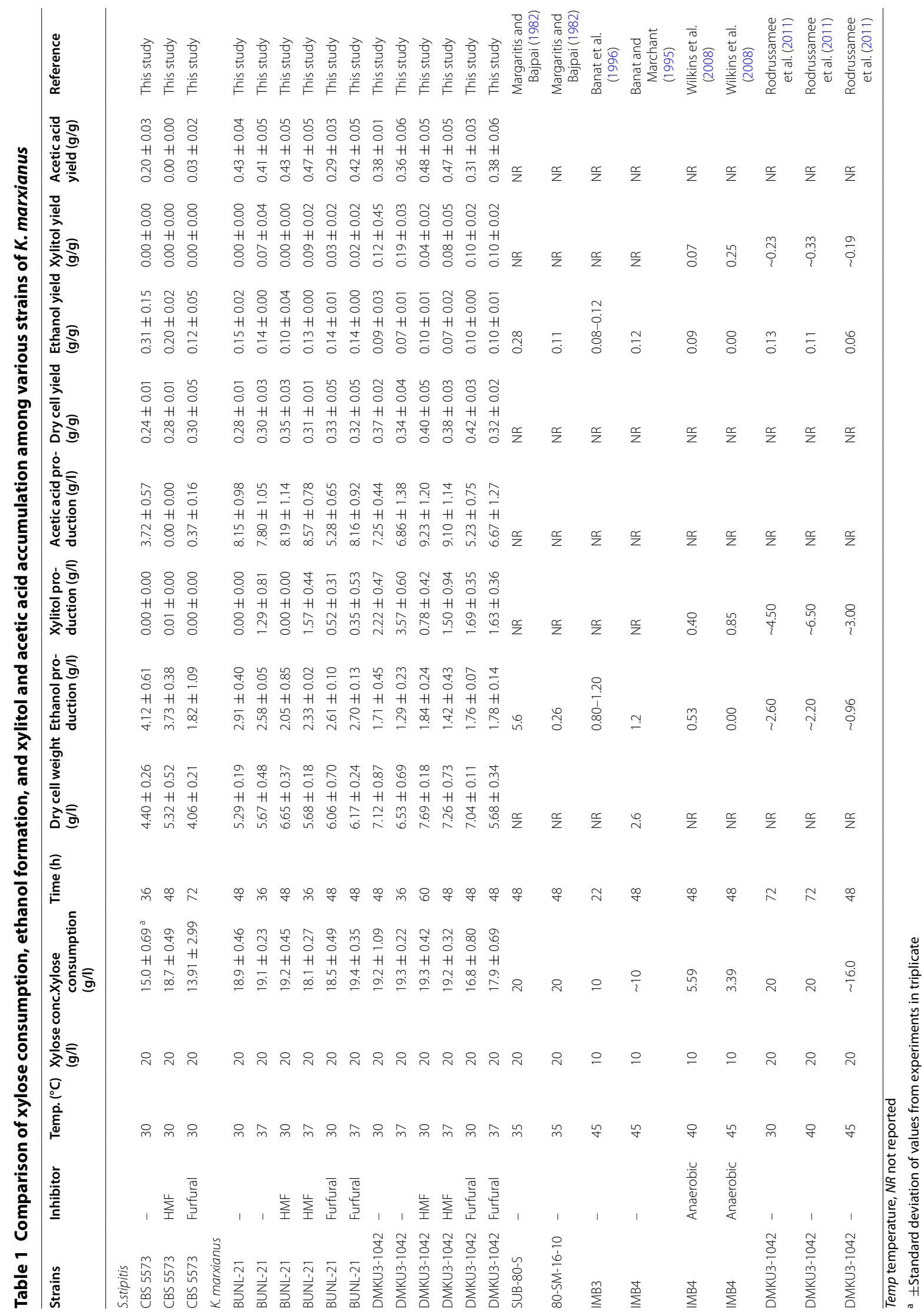


acid accumulation. Glycerol accumulation, however, was not detected in any of the strains tested (data not shown). These findings clearly indicate that the capability of conversion of Xyl to ethanol at a high temperature in $K$. marxianus is greater than that in S. stipitis and suggest that BUNL-21 is preferable to DMKU3-1042 for conversion at low and high temperatures.

\section{Stress resistance of $K$. marxianus BUNL-21 and DMKU3-1042 and S. stipitis}

During the fermentation process, yeast cells are exposed to several stresses including osmotic stress, ethanol stress and heat stress, all of which have severe effects on cell viability and ethanol production (Gibson et al. 2007; Puligundla et al. 2011). In addition, high temperature fermentation tends to generate oxidative stress inside cells (Zhang et al. 2015). Therefore, characteristics of not only efficient ethanol producibility but also tolerance to these stresses are potentially required for candidate yeasts for the ethanol fermentation industry.

The degree of stress tolerance of BUNL-21 was thus compared with those of DMKU3-1042 and S. stipitis.
Cells were spotted onto YPD agar plates supplemented with $5 \mathrm{mM} \mathrm{H}_{2} \mathrm{O}_{2}$ (oxidative stress), $35 \%$ Glc (osmotic stress) or $8 \%$ ethanol (ethanol stress), and effects of the supplements on cell growth were evaluated after incubation at $30{ }^{\circ} \mathrm{C}$ for $48 \mathrm{~h}$ (Fig. 2a). The three supplements were found to more strongly inhibit the growth of DMKU3-1042 and S. stipitis than that of BUNL-21.

The effects of furfural and HMF were also examined. The inhibitory effects of both compounds on the growth of BUNL-21 were nearly the same as those on the growth of DMKU3-1042. S. stipitis showed significantly weaker resistance than the two $K$. marxianus strains to furfural. BUNL-21 was also more resistant than DMKU3-1042 at $47^{\circ} \mathrm{C}$ (Fig. 2b). Taken together, the results clearly show that BUNL-21 is much more robust than DMKU3-1042 under the stress conditions tested.

\section{Effects of furfural and HMF on growth and ethanol fermentation on Xyl}

The effects of furfural and HMF on growth and ethanol fermentation on Xyl in K. marxianus BUNL-21, DMKU3-1042 and S. stipitis were further examined in

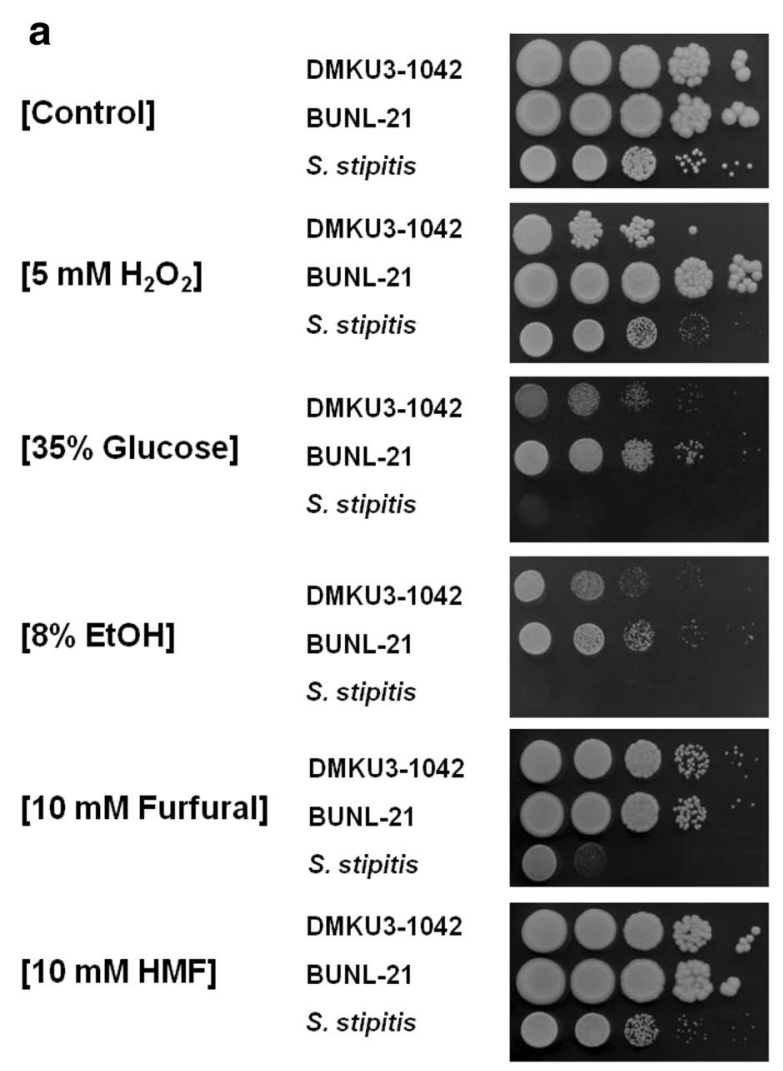

Fig. 2 Stress resistance of K. marxianus BUNL-21 and DMKU3-1042 and S. stipitis. Analysis of stress resistance was performed as described in "Methods". Diluted cells were spotted onto a YPD agar plates supplemented with $5 \mathrm{mM} \mathrm{H}_{2} \mathrm{O}_{2}, 8 \%$ ethanol, $35 \% \mathrm{Glc}, 10 \mathrm{mM}$ furfural or $10 \mathrm{mM} \mathrm{HMF}$. The plates were incubated at $30^{\circ} \mathrm{C}$ for $48 \mathrm{~h}$. b YPD agar medium was incubated at 30,45 or $47^{\circ} \mathrm{C}$ for $48 \mathrm{~h}$

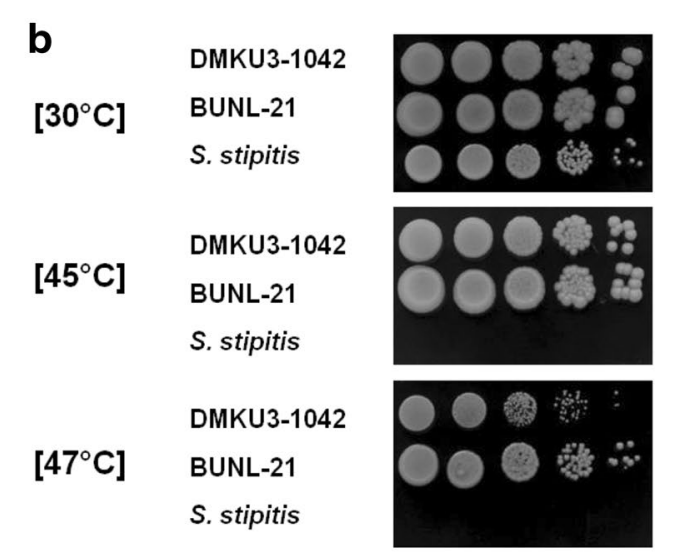


liquid media at 30 and $37{ }^{\circ} \mathrm{C}$ (Figs. 3, 4, Table 1). Study of S. stipitis at $37^{\circ} \mathrm{C}$ was omitted due to it being a nonpermissive temperature for this yeast (Fig. 1). Furfural and HMF showed strong or slightly negative effects, respectively, on growth and Xyl utilization of S. stipitis but hardly any or very slight negative effects on BUNL21 and DMKU3-1042. The timing of ethanol accumulation in the medium was retarded in the presence of the two compounds. At $30{ }^{\circ} \mathrm{C}$, the maximum ethanol yields of BUNL-21, DMKU3-1042 and S. stipitis were 0.10, 0.10 and $0.20 \mathrm{~g} / \mathrm{g}$, respectively, in YPXyl medium supplemented with $10 \mathrm{mM}$ HMF, and they were $0.14,0.10$ and $0.12 \mathrm{~g} / \mathrm{g}$, respectively, in the same medium supplemented with $10 \mathrm{mM}$ furfural. At $37{ }^{\circ} \mathrm{C}$, maximum ethanol yields of BUNL-21 and DMKU3-1042 were 0.13 and $0.07 \mathrm{~g} / \mathrm{g}$, respectively, in YPXyl medium supplemented with $10 \mathrm{mM}$ HMF, and they were 0.14 and $0.10 \mathrm{~g} / \mathrm{g}$, respectively, in the same medium supplemented with $10 \mathrm{mM}$ furfural. These findings and data shown in Fig. 2a suggest that both strains, especially BUNL-21, are relatively resistant to the typical toxic compounds derived from lingocellulosic biomass.

\section{Effect of 2-DOG on sugar utilization}

Glucose repression of the utilization of other sugars is one of the disadvantages for ethanol fermentation when biomass containing several sugars in addition to Glc is used. Thus, the effect of 2-DOG as an analog of Glc on sugar utilization was examined. Cells were spotted onto agar plates of YPGal, YPMan, YPXyl and YPAra with and without $0.01 \% 2$-DOG and were incubated at 30 and $37^{\circ} \mathrm{C}$ (Fig. 5). In the absence of 2-DOG, K. marxianus BUNL-21and DMKU3-1042 grew better on YPMan and YPGal than on YPXyl and YPAra at both temperatures. In the medium supplemented with $0.01 \%$ 2-DOG, the growth on YPGal, YPXyl and YPAra, but not that on YPMan, significantly decreased. The inhibitory effect of 2-DOG on DMKU3-1042, but not that on BUNL-21, was slightly enhanced with an increase in temperature. In addition, BUNL-21 showed better growth than that of DMKU3-1042 on YPXyl and YPAra plates supplemented with 2-DOG. These results suggest that $K$. marxianus BUNL-21 exhibits a relatively weak glucose repression, especially of Xyl or Ara utilization, and is thus more suitable than DMKU31042 for application of lignocellulosic biomass.

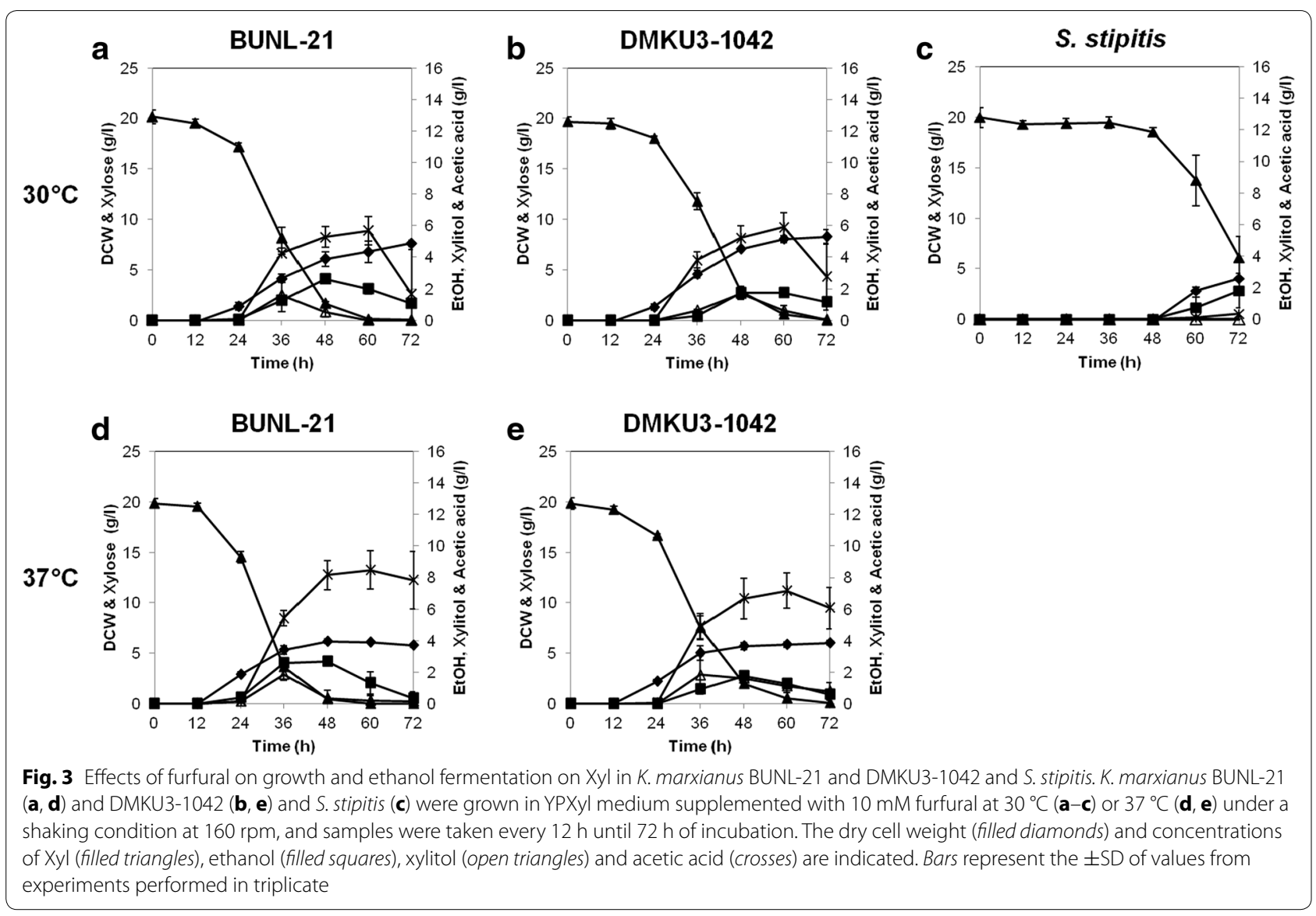




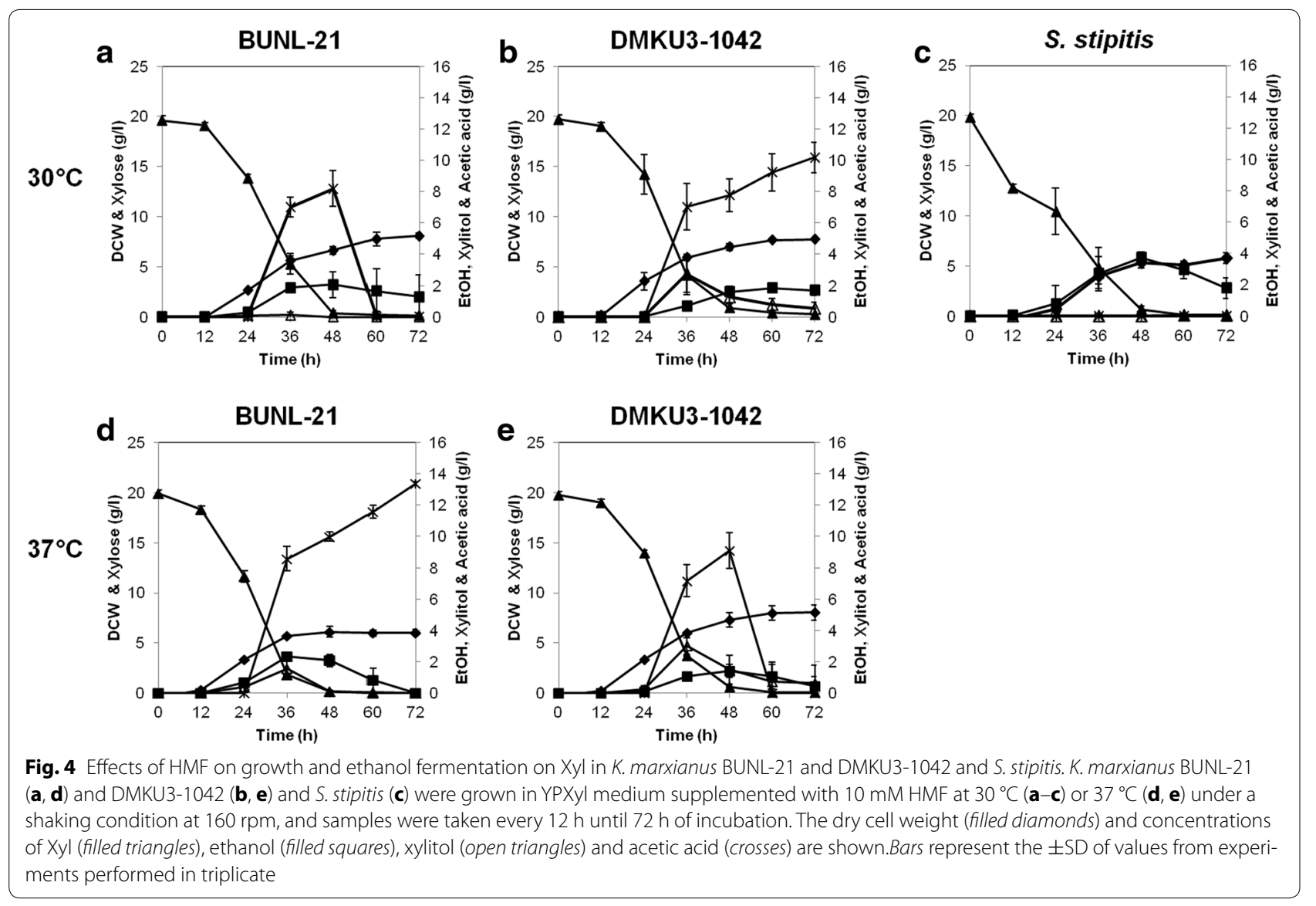

\section{Effect of Glc on Xyl utilization}

To further analyze the glucose repression of $K$. marxianus BUNL-21 and DMKU3-1042, effects of Glc on their Xyl utilization were examined (Fig. 6). Consumption patterns of Glc in the presence of Xyl were similar to those in the absence of Xyl (data not shown), and Glc in 0.2 and $2 \%$ Glc YP media was completely used within $12 \mathrm{~h}$. On the other hand, when Xyl consumption with and that without $2 \%$ Glc in YPXyl medium were compared, the utilization of Xyl was greatly delayed, especially in DMKU3-1042, in the presence of Glc (Figs. 1, 6). Consistently, in the YP medium of $2 \% \mathrm{Xyl}+0.2 \%$ Glc, a relatively strong effect on Xyl consumption was observed in DMKU3-1042, but the effect was relatively weak in BUNL-21, indicating that BUNL-21 consumed Xyl faster than did DMKU31042 both at low and high Glc concentrations. These results seem to agree with the finding that BUNL21 exhibited weaker glucose repression than that of DMKU3-1042 in Xyl plates as shown in Fig. 5. Moreover, the maximum ethanol concentration of BUNL-21 in the mixed sugar medium was higher than that of DMKU3-1042.

\section{Discussion}

In this study, we compared the capability for conversion of Xyl to ethanol of the thermotolerant $K$. marxianus strain BUNL-21 from Laos with that of the efficient strain DMKU3-1042 from Thailand and S. stipitis. Comparison of the ethanol productivity from Xyl or/and Glc (Figs. 1, 6), degrees of tolerance to various stresses (Figs. 2, 3, 4) and degree of 2-DOG tolerance indicating susceptibility to glucose repression (Figs. 2, 5) suggests that BUNL-21 has a high potential that is superior to that of DMKU3-1042 for application in ethanol production from lignocellulosic biomass. In addition, we noticed accumulation of xylitol and large accumulation of acetic acid over ethanol in $K$. marxianus but not in S. stipitis when Xyl was used as a carbon source. The accumulation of acetic acid seems to result in a low level of ethanol production in K. marxianus.

$K$. marxianus and S. stipitis are Crabtree-negative yeasts. Several studies have revealed that $K$. marxianus is superior to $S$. stipitis in thermotolerance and stress resistance but inferior in terms of ethanol productivity from Xyl and oxygen dependence (Jeffries and Van Vleet 2009; Lertwattanasakul et al. 2015; Signori et al. 2014). S. stipitis CBS5773 and CBS 6054 achieved ethanol yields of 


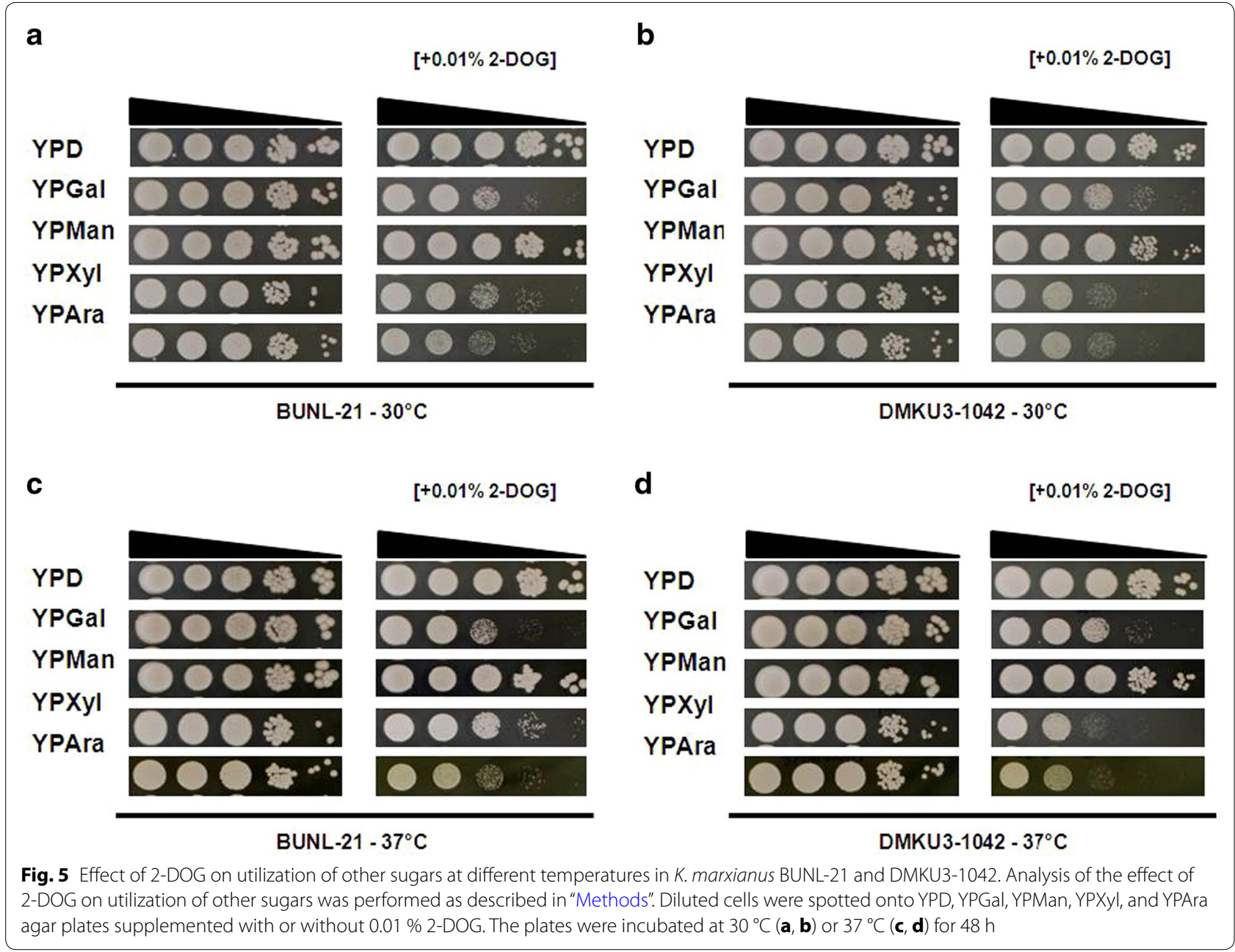

$\sim 0.41 \mathrm{~g} / \mathrm{g}$ under both anaerobic and microaerobic conditions (Krahulec et al. 2012). Under the conditions used in this study, S. stipitis CBS 5773 achieved ethanol yield of $0.31 \mathrm{~g} / \mathrm{g}$ at $30{ }^{\circ} \mathrm{C}$. On the other hand, K. marxianus DMKU3-1042 achieved ethanol yields of 0.13, 0.11 and $0.06 \mathrm{~g} / \mathrm{g}$ at $30^{\circ} \mathrm{C}, 40$ and $45^{\circ} \mathrm{C}$, respectively, under a shaking condition, but its ethanol yield was negligible under a static condition (Rodrussamee et al. 2011). K. marxianus IMB4 showed ethanol yields of $\sim 0.12 \mathrm{~g} / \mathrm{g}$ at $45^{\circ} \mathrm{C}$ (Banat and Marchant 1995) under an aerobic condition, but its ethanol yield decreased to $0.09 \mathrm{~g} / \mathrm{g}$ at $40{ }^{\circ} \mathrm{C}$ and $0.00 \mathrm{~g} / \mathrm{g}$ at $45^{\circ} \mathrm{C}$ under an anaerobic condition (Banat and Marchant 1995; Wilkins et al. 2008). These results suggest that $K$. marxianus has a higher oxygen dependency than that of S. stipitis.

As described above, the thermotolerant characteristics of $K$. marxianus are beneficial for high-temperature fermentation or robust fermentation. The two $K$. marxianus strains tested in this study were shown to be able to ferment $\mathrm{Xyl}$ at $37{ }^{\circ} \mathrm{C}$, a temperature at which S. stipitis cannot grow. The yield of ethanol production from Xyl of $K$. marxianus varies in different strains from 0.1 to $0.28 \mathrm{~g} / \mathrm{g}$ (Margaritis and Bajpai 1982; Banat et al. 1996; Wilkins et al. 2008; Rodrussamee et al. 2011) (Table 1). However, BUNL-21 achieved ethanol yields of 0.15 and $0.14 \mathrm{~g} / \mathrm{g}$ on Xyl at 30 and $37{ }^{\circ} \mathrm{C}$, respectively, which were about 1.7 - and 2.0-times higher, respectively, than those of DMKU31042 (Table 1). Regarding ethanol yield, although the fermentation conditions were not the same, BUNL21 showed ethanol yields higher than those of strains 80-SM-16-10, IBM3 and IBM4, the ethanol yields of which were $0.11,0.12$ and $0.12 \mathrm{~g} / \mathrm{g}$, respectively, but lower than that of strain SUB-80-S, the ethanol yield of which was $0.28 \mathrm{~g} / \mathrm{g}$ at $35^{\circ} \mathrm{C}$ (Banat and Marchant 1995; Banat et al. 1996; Margaritis and Bajpai 1982). Moreover, it is notable that the productivity of both strains was hardly inhibited by HMF or furfural, and the ethanol yield of BUNL-21 was $0.14 \mathrm{~g} / \mathrm{g}$ in the presence of furfural (Figs. 3, 4, Table 1). 


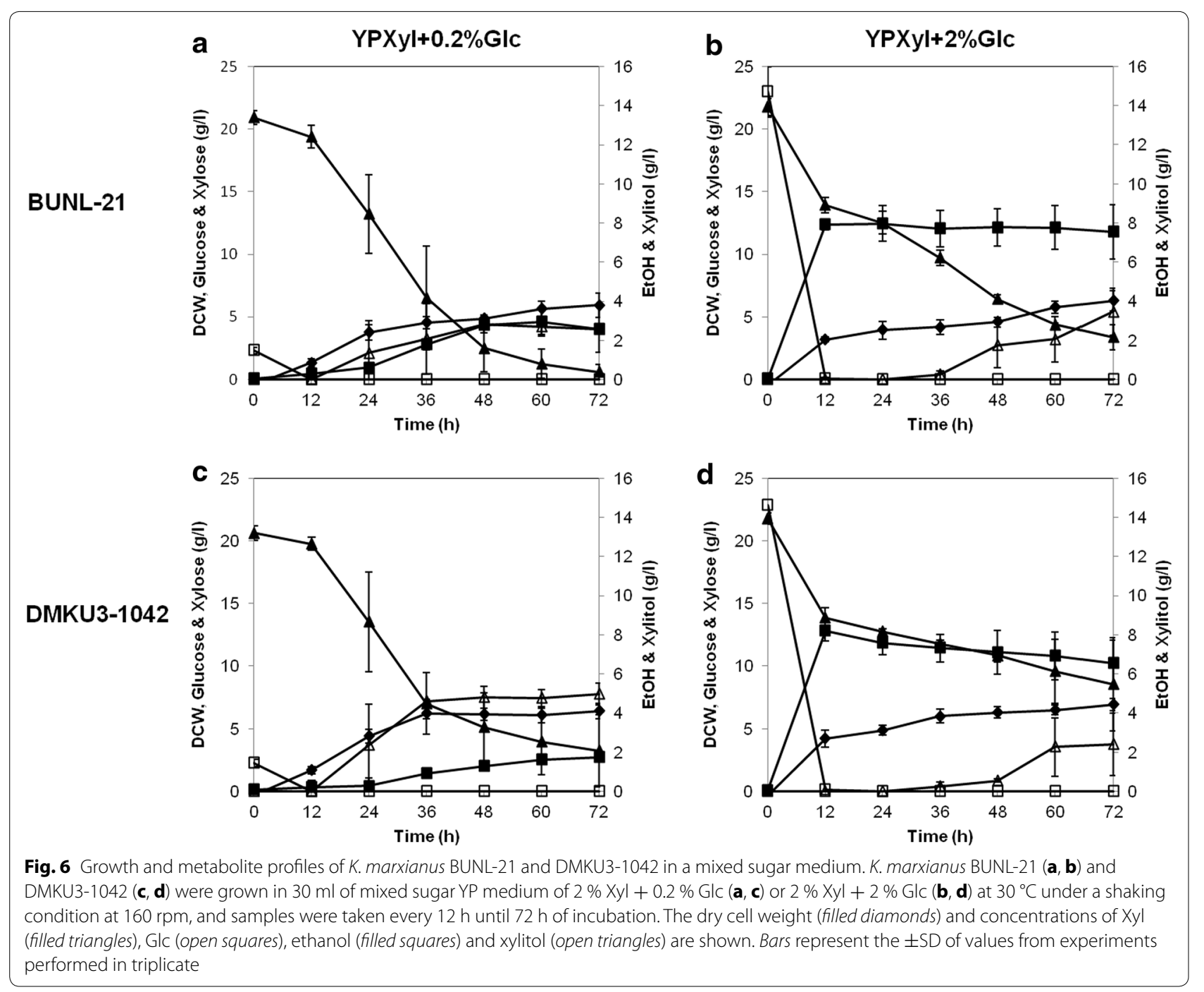

It was found that both $K$. marxianus strains accumulated larger amounts of xylitol than that accumulated by $S$. stipitis. The accumulation of xylitol seems to reduce the yield of ethanol. We have limited evidence regarding the superiority of BUNL-21 to DMKU3-1042. BUNL-21 accumulated a smaller amount of xylitol both at 30 and $37^{\circ} \mathrm{C}$ (Fig. 1). Consistently, when xylitol was used as a carbon source, BUNL-21 assimilated xylitol and grew better than DMKU3-1042 did (Additional file 1: Fig. S2). Notably, the expression level of $A D H 2$, which is a gene for the major alcohol dehydrogenase in ethanol fermentation on Glc (Lertwattanasakul et al. 2007) and is down-regulated on Xyl (Lertwattanasakul et al. 2015), in BUNL-21 was found to be about 3-times higher than that in DMKU31042 on Xyl (Additional file 1: Fig. S3). Its relatively high expression level of $A D H 2$ may provide more $\mathrm{NAD}^{+}$to xylitol dehydrogenase to prevent xylitol accumulation and produce more ethanol. Moreover, it was found that a higher temperature caused more accumulation of xylitol in both $K$. marxianus strains (Fig. 1). The accumulation might be due to the limitation of $\mathrm{NAD}^{+}$as a result of the so-called cofactor imbalance (Jeffries and Jin 2004; Zhang et al. 2013; Hou et al. 2014). If so, it is assumed that some enzyme activity coupled to NADH oxidation is weakened at a high temperature. Alternatively, the pathway from Xyl uptake to xylitol might be enhanced or the pathway downstream from xylitol might be weakened as temperature increased.

Large accumulation of acetic acid (0.36-0.43 g/g) was found in both $K$. marxianus strains at 30 and $37{ }^{\circ} \mathrm{C}$, which may be responsible for the low level of ethanol production. One speculative reason for the acetic acid accumulation is the requirement of NADPH for xylose reductase in xylose catabolism (Signori et al. 2014). The 
accumulation is consistent with the up-regulation of ALD4 for acetaldehyde dehydrogenase on Xyl (Fig. 7) (Lertwattanasakul et al. 2015). At the same time, RKI1 for ribose-5-phosphate isomerase in the pentose phosphate pathway (PPP) is largely down-regulated (Fig. 7). We thus speculated that the NADPH supply for xylose reductase activity by PPP is limited and compensatorily the cofactor is provided by the acetaldehyde dehydrogenase-mediated reaction. On the other hand, no such down-regulation of any of the genes including RKI1 for PPP occurs in S. stipitis (Jeffries and Van Vleet 2009). However, when xylulose was used as a carbon source, the level of acetic acid was hardly changed (Additional file 1: Fig. S4). Another possible reason is the supply of NADPH for removal of reactive oxygen species (ROS) via antioxidants including glutathione. Evidence that genes for the oxidative stress defense mechanism were up-regulated in Xyl medium (Lertwattanasakul et al. 2015) indicates the possibility that $K$. marxianus accumulates ROS under the condition with Xyl.

Notably, in Glc medium, large accumulation of acetic acid was observed at $45{ }^{\circ} \mathrm{C}$, but not at $30{ }^{\circ} \mathrm{C}$, and the accumulation of acetic acid was prevented by the addition of the reduced form of glutathione (unpublished data). These findings allow us to speculate that cells accumulate ROS at a high temperature and, via the acetic acid production pathway, supply NADPH for detoxification of ROS. In Xyl medium, however, the addition of reduced glutathione showed no effect on the accumulation of acetic acid. This failure might be due to the lower expression levels of genes for the putative glutathione transporters (OPT1 and OPT2) in Xyl medium (Lertwattanasakul et al. 2015). Another possible reason for the failure is that cells require a much larger amount of NADPH for other cellular activities under the condition with $\mathrm{Xyl}$ as a carbon source than those with Glc at $45^{\circ} \mathrm{C}$.

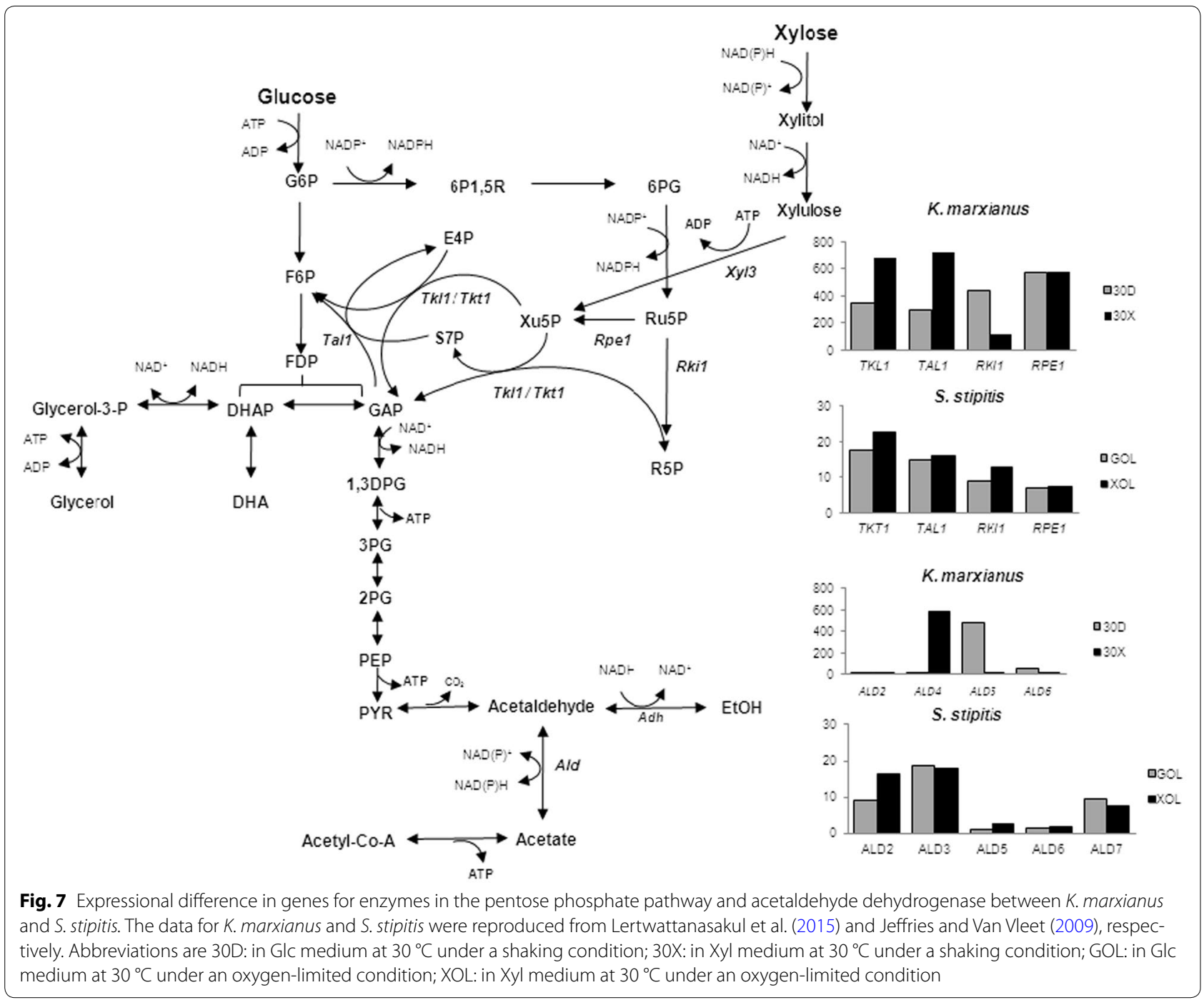




\section{Conclusion}

Application of a stress-resistant and highly efficient microbe for ethanol fermentation is a crucial point for industrial application. This study provided evidence that the newly isolated strain of $K$. marxianus BUNL-21 bears a high potential for conversion of Xyl to ethanol, has strong resistance to high temperature and to toxic materials, and exhibits relatively weak glucose repression. These beneficial characteristics will allow us to develop a more efficient Xyl-to-ethanol converter by gene engineering on the basis of BUNL-21. The first findings of a large accumulation of acetic acid and its relation to specific gene expression on Xyl in K. marxianus motivate us to do gene engineering to improve NADPH production and reduce acetic acid accumulation.

\section{Additional file}

Additional file 1: Fig. S1. Relation of dry cell weight to $\mathrm{OD}_{660}$ of $K$. marxianus BUNL-21 and DMKU3 1042 and S. stipitis. Fig. S2. Growth and metabolite profiles of K. marxianus BUNL-21 and DMKU3-1042 in YP medium containing $2 \%$ xylitol. Fig. S3. Expression of $K m A D H 1$ and $\mathrm{KmADH} 2$ genes of $K$. marxianus BUNL-21 and DMKU3-1042 in YPXYl medium. Fig. S4. Growth and metabolite profiles of K. marxianus BUNL-21 in YP medium containing $2 \%$ xylulose.

\section{Authors' contributions}

SN performed almost wet lab experiments and writing of the manuscript. CK and SL provided K. marxianus BUNL-21 and DMKU3-1042, respectively, and their fundamental information. MM, NL and TK provided technical supports and participated in discussion of this study. MY involved in the design and discussion of this study and prepared the manuscript. All authors read and approved the final manuscript.

\section{Author details \\ 1 Applied Molecular Bioscience, Graduate School of Medicine, Yamaguchi University, Ube 755-8505, Japan. ${ }^{2}$ Department of Biology, Faculty of Science, National University of Laos, Vientiane, Lao PDR. ${ }^{3}$ Department of Microbiology, Faculty of Science, Kasetsart University, Bangkok 10900, Thailand. ${ }^{4}$ Depart- ment of Biological Chemistry, Faculty of Agriculture, Yamaguchi University, Yamaguchi 753-8515, Japan.}

\section{Acknowledgements}

We thank Drs. Matsushita and Yakushi for their helpful discussions. This work was supported by the Special Coordination Funds for Promoting Science and Technology, Ministry of Education, Culture, Sports, Science and Technology (MEXT), and the Advanced Low Carbon Technology Research and Development Program, Japan Science and Technology Agency (JST). This work was partially performed as collaborative research in the Asian Core Program and in the Core to Core Program, which was supported by the Scientific Cooperation Program agreed by the Japan Society for the Promotion of Science (JSPS), the National Research Council of Thailand (NRCT) and universities involved in the program.

\section{Competing interests}

All authors declare that they have no competing interests.

\section{Ethical approval}

This article does not contain any studies with human participants or animals performed by any of the authors.

Received: 28 September 2015 Accepted: 16 February 2016

Published online: 27 February 2016

\section{References}

Anderson PJ, McNeil K, Watson K (1986) High-efficiency carbohydrate fermentation to ethanol at temperatures above $40^{\circ} \mathrm{C}$ by Kluyveromyces marxianus var. marxianus isolated from sugar mills. Appl Environ Microbiol 51:1314-1320

Banat IM, Marchant R (1995) Characterization and potential industrial applications of five novel, thermotolerant, fermentative, yeast strains. World J Microbiol Biotechnol 11:304-306

Banat IM, Singh D, Marchant R (1996) The use of a thermotolerant fermentative Kluyveromyces marxianus IMB3 yeast strain for ethanol production. Acta Biotechnol 16:215-223

Banat IM, Nigam P, Singh D, Marchantand R, McHale AP (1998) Ethanol production at elevated temperatures and alcohol concentrations: part l-yeasts in general. World J Microbiol Biotechnol 14:809-821

Barnett JA, Payne RW, Yarrow D (2000) Yeasts: characteristics and identification, 3rd edn. Cambridge University Press, Cambridge

Behera S, Arora R, Nandhagopal N, Kumar S (2014) Importance of chemical pretreatment for bioconversion of lignocellulosic biomass. Renew Sustain Energy Rev 36:91-106. doi:10.1016/j.rser.2014.04.047

Chain EB, Gualandi G (1954) Aeration studies II. Rend Ist Super Sanita (English Ed) 17:5-60

Gibson BR, Lawrence SJ, Leclaire JPR, Powell CD, Smart KA (2007) Yeast responses to stresses associated with industrial brewery Handling. FEMS Microbiol Rev 31:535-569. doi:10.1111/j.1574-6976.2007.00076.x

Hou J, Suo F, Wang C, Li X, Shen Y, Bao X (2014) Fine-tuning of NADH oxidase decreases byproduct accumulation in respiration deficient xylose metabolic Saccharomyces cerevisiae. BMC Biotechnol 14:13. doi:10.1186/1472-6750-14-13

Jeffries TW, Jin YS (2004) Metabolic engineering for improved fermentation of pentoses by yeasts. Appl Microbiol Biotechnol 63:495-509. doi:10.1007/ s00253-003-1450-0

Jeffries TW, Van Vleet JR (2009) Pichia stipitis genomics, transcriptomics, and gene clusters. FEMS Yeast Res 9:793-807. doi:10.1111/j.1567-1364.2009.00525.x

Krahulec S, Kratzer R, Longus K, Nidetzky B (2012) Comparison of Scheffersomyces stipitis strains CBS 5773 and CBS 6054 with regard to their xylose metabolism: implications for xylose fermentation. Microbiologyopen 1(1):64-70. doi:10.1002/mbo3.5

Kurtzman CP, Fell JW, Boekhout T (2011) The yeasts: a taxonomic study, 5th edn. Elsevier, Amsterdam

Lertwattanasakul N, Sootsuwan K, Limtong S, Thanonkeo P, Yamada M (2007) Comparison of the gene expression patterns of alcohol dehydrogenase isozymes in the thermotolerant yeast Kluyveromyces marxianus and their physiological functions. Biosci Biotechnol Biochem 71:1170-1182

Lertwattanasakul N, Rodrussamee N, Suprayogi Limtong S, Thanonkeo P, Kosaka T, Yamada M (2011) Utilization capability of sucrose, raffinose and inulin and its less-sensitiveness to glucose repression in thermotolerant yeast Kluyveromyces marxianus DMKU3-1042. AMB Express 1:20

Lertwattanasakul N, Kosaka T, Hosoyama A, Suzuki Y, Rodrussamee N, Matsutani M, Murata M, Fujimoto N, Suprayogi Tsuchikane K, Limtong S, Fujita N, Yamada M (2015) Genetic basis of the highly efficient yeast Kluyveromyces marxianus: complete genome sequence and transcriptome analyses. Biotechnol Biofuels 8:47. doi:10.1186/s13068-015-0227-x

Limtong S, Sringiew C, Yongmanitchai W (2007) Production of fuel ethanol at high temperature from sugar cane juice by a newly isolated Kluyveromyces marxianus. Bioresour Technol 98:3367-3374. doi:10.1016/j. biortech.2006.10.044

Margaritis A, Bajpai P (1982) Direct fermentation of D-xylose to ethanol by Kluyveromyces marxianus strains. Appl Environ Microbiol 44(5):1039-1041

Murata M, Nitiyon S, Lertwattanasakul N, Sootsuwan K, Kosaka T, Thanonkeo P, Limtong S, Yamada M (2015) High-temperature fermentation technology for low cost bioethanol. J Jpn Inst Energy 94:1154-1162

Mussatto SI, Roberto IC (2004) Alternatives for detoxification of diluted-acid lignocellulosic hydrolyzates for use in fermentative processes: a review. Bioresour Technol 93:1-10. doi:10.1016/j.biortech.2003.10.005

Nguyen NH, Suh SO, Marshall CJ, Blackwell M (2006) Morphological and ecological similarities: wood-boring beetles associated with novel xylose-fermenting yeasts, Spathaspora passalidarum gen. sp. nov. and Candida jeffriesii sp. nov. Mycol Res 110:1232-1241. doi:10.1016/j. mycres.2006.07.002 
Nigam JN (2001) Ethanol production from wheat straw hemicelluloses hydrolysate by Pichia stipitis. J Biotechnol 87:17-27. doi:10.1016/ S0168-1656(00)00385-0

Pimpakan P, Yongmanitchai W, Limtong S (2012) Bioethanol production from sugar cane syrup by thermo-tolerant yeast, Kluyveromyces marxianus DMKU3-1042, using fed-batch and repeated-batch fermentation in a nonsterile system. Kasetsart J (Nat Sci) 46:582-591

Puligundla P, Smogrovicova D, Obulam VSR, Ko S (2011) Very high gravity (VHG) ethanolic brewing and fermentation: a research update. J Ind Microbiol Biotechnol 38:1133-1144. doi:10.1007/s10295-011-0999-3

Rodrussamee N, Lertwattanasakul N, Hirata K, Suprayogi Limtong S, Kosaka T, Yamada M (2011) Growth and ethanol fermentation ability on hexose and pentose sugars and glucose effect under various conditions in thermotolerant yeast Kluyveromyces marxianus. Appl Microbiol Biotechnol 90:1573-1586. doi:10.1007/s00253-011-3218-2

Signori L, Passolunghi S, Ruohonen L, Porro D, Branduardi P (2014) Effect of oxygenation and temperature on glucose-xylose fermentation in Kluyveromyces marxianus CBS712 strain. Microb Cell Fact 13:51. doi:10.1186/1475-2859-13-51

Su YK, Willis LB, Jeffries TW (2015) Effects of aeration on growth, ethanol and polyol accumulation by Spathaspora passalidarum NRRLY-27907 and Scheffersomyces stipitis NRRL Y-7124. Biotechnol Bioeng 112(3):457-469. doi:10.1002/bit.25445
Toivola A, Yarrow D, van den Bosch E, van Dijken JP, Scheffers WA (1984) Alcoholic fermentation of D-xylose by yeasts. Appl Environ Microbiol 47:1221-1223

Wang R, Li L, Zhang B, Gao X, Wang D, Hong J (2013) Improved xylose fermentation of Kluyveromyces marxianus at elevated temperature through construction of a xylose isomerase pathway. J Ind Microbiol Biotechnol 40:841-854. doi:10.1007/s10295-013-1282-6

Wilkins MR, Mueller M, Eichling S, Banat IM (2008) Fermentation of xylose by the thermotolerant yeast strains Kluyveromyces marxianus IMB2, IMB4, and IMB5 under anaerobic conditions. Process Biochem 43(4):346-350. doi:10.1016/j.procbio.2007.12.011

Zhang B, Li L, Zhang J, Gao X, Wang D, Hong J (2013) Improving ethanol and xylitol fermentation at elevated temperature through substitution of xylose reductase in Kluyveromyces marxianus. J Ind Microbiol Biotechnol 40:305-316. doi:10.1007/s10295-013-1230-5

Zhang M, Shi J, Jiang L (2015) Modulation of mitochondrial membrane integrity and ROS formation by high temperature in Saccharomyces cerevisiae. Electron J Biotechnol. doi:10.1016/j.ejbt.2015.03.008

\section{Submit your manuscript to a SpringerOpen ${ }^{\circ}$ journal and benefit from:}

- Convenient online submission

- Rigorous peer review

- Immediate publication on acceptance

- Open access: articles freely available online

- High visibility within the field

- Retaining the copyright to your article

Submit your next manuscript at $>$ springeropen.com 